\title{
Coherent and incoherent chaotic tunneling near singlet-doublet crossings
}

\author{
Sigmund Kohler, Ralf Utermann, and Peter Hänggi \\ Institut für Physik, Universität Augsburg, Memminger Straße 6, D-86135 Augsburg, Germany \\ Thomas Dittrich* \\ Max-Planck-Institut für Physik komplexer Systeme, Nöthnitzer Straße 38, D-01187 Dresden, \\ Germany
}

(June 30, 2021)

\begin{abstract}
In the spectrum of systems showing chaos-assisted tunneling, three-state crossings are formed when a chaotic singlet intersects a tunnel doublet. We study the dissipative quantum dynamics in the vicinity of such crossings. A harmonically driven double well coupled to a bath serves as a model. Markov and rotating-wave approximations are introduced with respect to the Floquet spectrum of the time-dependent central system. The resulting master equation is integrated numerically. We find various types of transient tunneling, determined by the relation of the level width to the inherent energy scales of the crossing. The decay of coherent tunneling can be significantly retarded or accelerated. Modifications of the quantum asymptotic state by the crossing are also studied. The comparison with a simple three-state model shows that in contrast to the undamped case, the participation of states outside the crossing cannot be neglected in the presence of dissipation.
\end{abstract}

05.30.-d, 42.50.Lc, 03.65.Sq

Typeset using REVTEX

*Present address: Division de Physique Théorique, Institut de Physique Nucléaire, F-91406 Orsay Cedex, France 


\section{INTRODUCTION}

In a quantum setting, the coexistence of regular and chaotic regions in a mixed phase space leads to a variety of uncommon coherence phenomena. Most prominent among them is chaotic tunneling [1- 14], the coherent exchange of probability between symmetry-related regular islands that are separated by a chaotic layer, not by a static potential barrier. Chaotic tunneling comes about by an interplay of classical nonlinear - typically bistable - dynamics and quantum coherence. It therefore reflects features of the classical phase space, such as the width of the chaotic layer [7,8] and the structure of the "coast line" [11,12] separating it from the adjacent regular regions, as well as fine details of the quantum spectrum like exact and avoided crossings. In previous work [7,8], it has been shown that, as the chaotic layer grows with increasing nonlinearity, the tunnel splittings widen collectively. Superimposed on this global trend of the parameter dependence, however, there are strong fluctuations, occurring on a smaller parameter scale and restricted to individual tunnel doublets [6, 11, 13, 14]. A major source of these fluctuations are the disturbances of doublets, suffered as they are intersected by other levels. The most common type of such intersections is formed when a doublet, pertaining to a pair of eigenstates located on symmetry-related quantizing tori, encounters a singlet that belongs to an eigenstate in the chaotic sea [10]. The chaotic states, even if they no longer come in pairs close in energy, can still be classified as even or odd. This fact determines the structure of the singlet-doublet crossings: The partner in the doublet sharing the symmetry of the chaotic singlet is repelled by the singlet-together, they form an avoided crossing. The partner with opposite symmetry must either be intersected in an exact crossing close to the avoided one, or else the order within the doublet is reversed from one side of the crossing to the other. The variety of configurations of such crossings is sketched in Fig. 1.

In the present paper, we study chaotic tunneling in the vicinity of singlet-doublet crossings, under the influence of incoherent processes. Near a crossing, level separations deviate vastly, in both directions, from the typical tunnel splitting (cf. Fig. 1). This is reflected in time-domain phenomena ranging from the suppression of tunneling to a strong increase in its rate and to complicated quantum beats [10]. In Section II], we briefly review chaotic tunneling in our working model, a harmonically driven quartic double well [15 [17. Singletdoublet crossings are identified and characterized by their signature in terms of quasienergy and mean energy. We describe the behaviour of the eigenstates close to a crossing and analyze the coherent dynamics in terms of a simple three-state model.

Tunneling is associated with extremely small energy scales, all the more in the semiclassical regime we are interested in. It is therefore particularly sensitive to any disruption of coherence as it occurs due to the unavoidable coupling to the environment. As immediate consequences, the symmetry underlying the formation of tunnel doublets is generally broken, and an additional energy scale is introduced, the effective finite width attained by each discrete level. Tunneling and related coherence phenomena are thus rendered transients that occur - if at all — on the way towards an asymptotic equilibrium state. Singlet-doublet cross-

ings, in turn, drastically change the nondissipative energy scales and replace the two-level by a three-level structure. As a consequence, the familiar way tunneling fades out in the presence of dissipation is also significantly altered. Near a crossing, the coherent dynamics can last much longer than for the unperturbed doublet, despite the presence of the same 
decoherence than outside the crossing, establishing "chaos-induced coherence". Depending on temperature, it can also be destroyed on a much shorter time scale.

In Section [II], we first outline the modifications of our model necessary to incorporate dissipation. We treat the quantum dissipative dynamics within the framework of the Floquet formalism [18 23]. In the present context of an interplay of tunneling with incoherent processes, we focus on the regime of weak dissipation. Very long time scales are therefore involved, both in the coherent dynamics and in the bath response. In this case, the usual (Markov and rotating-wave) approximations resorted to in the elimination of the external degrees of freedom, have to be reconsidered critically. The coupling to the environment indirectly couples the three levels in the crossing to all the other states of the central system. On the basis of numerical results for the full driven double well with dissipation, we reveal the limitations of the three-level approximation and identify additional features of the full dynamics not covered by it. In particular, we consider the long-time asymptotics - the quantum attractor - and the phase-space structure associated with it. Section $\mathbb{\text { IV }}$ serves to summarize our results and to suggest directions of further research.

\section{CONSERVATIVE CLASSICAL AND QUANTUM DYNAMICS}

\section{A. The model and its symmetries}

As a prototypical working model, we consider the quartic double well with a spatially homogeneous driving force harmonic in time. It is defined by the Hamiltonian

$$
\begin{aligned}
H_{\mathrm{DW}}(x, p ; t) & =H_{0}(x, p)+H_{1}(x ; t), \\
H_{0}(x, p) & =\frac{p^{2}}{2}-\frac{1}{4} x^{2}+\frac{1}{64 D} x^{4}, \\
H_{1}(x ; t) & =S x \cos (\omega t) .
\end{aligned}
$$

Apart from mere scaling, the classical phase space of $H_{0}(x, p)$ depends only on the presence or absence, and the sign, of the $x^{2}$ term. Otherwise, it has no free parameter. In the quantummechanical case, the parameter $D / \hbar$ plays the rôle of an inverse quantum of action. It controls the barrier height and can be interpreted as the (approximate) number of doublets with energies below the top of the barrier. Accordingly, the classical limit is reached by letting $D / \hbar \rightarrow \infty$. The influence of the driving on the classical phase space structure is characterized by the rescaled amplitude

$$
F=S / \sqrt{8 D}
$$

and frequency $\omega$. This implies that the classical dynamics is independent of the barrier height $D$.

The unperturbed Hamiltonian $H_{0}(x, p)$ is invariant under the parity $\mathrm{P}: x \rightarrow-x, p \rightarrow-p$, $t \rightarrow t$. This symmetry is generally destroyed by the driving. With the above choice of $H_{1}(x ; t)$, however, a more general, dynamical symmetry is retained [15 17,24]. It is defined by the operation

$$
\mathrm{P}_{\omega}: \quad x \rightarrow-x, \quad p \rightarrow-p, \quad t \rightarrow t+\pi / \omega
$$


and represents a generalized parity acting in the extended phase space spanned by $x, p$, and phase, i.e., time $t \bmod (2 \pi / \omega)$. The invariance under $\mathrm{P}_{\omega}$ of $H(x, p ; t)$ allows to classify all its quantum eigenstates as even or odd.

As a consequence of the periodic time dependence of $H(x, p ; t)$, the relevant generator of the quantum dynamics is now the Floquet operator 25 29.

$$
U=\mathcal{T} \exp \left(-\frac{\mathrm{i}}{\hbar} \int_{0}^{2 \pi / \omega} \mathrm{d} t H(t)\right)
$$

where $\mathcal{T}$ denotes time ordering. According to the Floquet theorem, the adiabatic states of the system are the eigenstates of $U$. They can be written in the form

$$
\left|\psi_{\alpha}(t)\right\rangle=\mathrm{e}^{-\mathrm{i} \epsilon_{\alpha} t / \hbar}\left|\phi_{\alpha}(t)\right\rangle
$$

with

$$
\left|\phi_{\alpha}(t+2 \pi / \omega)\right\rangle=\left|\phi_{\alpha}(t)\right\rangle .
$$

Expanded in the basis spanned by these Floquet states, the propagator of the driven system reads

$$
U\left(t^{\prime}, t\right)=\sum_{\alpha} \mathrm{e}^{-\mathrm{i} \epsilon_{\alpha}\left(t^{\prime}-t\right) / \hbar}\left|\phi_{\alpha}\left(t^{\prime}\right)\right\rangle\left\langle\phi_{\alpha}(t)\right|
$$

The associated eigenphases $\epsilon_{\alpha}$, referred to as quasienergies, come in classes, $\epsilon_{\alpha, n}=\epsilon_{\alpha}+n \hbar \omega$, $n=0, \pm 1, \pm 2, \ldots$. This is suggested by a Fourier expansion of the $\left|\phi_{\alpha}(t)\right\rangle$,

$$
\begin{aligned}
\left|\phi_{\alpha}(t)\right\rangle & =\sum_{n}\left|c_{\alpha, n}\right\rangle \mathrm{e}^{-\mathrm{i} n \omega t}, \\
\left|c_{\alpha, n}\right\rangle & =\frac{\omega}{2 \pi} \int_{0}^{2 \pi / \omega} \mathrm{d} t\left|\phi_{\alpha}(t)\right\rangle \mathrm{e}^{\mathrm{i} n \omega t} .
\end{aligned}
$$

The index $n$ counts the number of quanta in the driving field. Otherwise the members of a class $\alpha$ are physically equivalent. Therefore, the quasienergy spectrum can be reduced to a single "Brillouin zone", $-\hbar \omega / 2 \leq \epsilon<\hbar \omega / 2$.

Since the quasienergies have the character of phases, they can be ordered only locally, not globally. A quantity that is defined on the full real axis and therefore does allow for a complete ordering, is the mean energy [28,29]

$$
E_{\alpha}=\frac{\omega}{2 \pi} \int_{0}^{2 \pi / \omega} \mathrm{d} t\left\langle\psi_{\alpha}(t)|H(t)| \psi_{\alpha}(t)\right\rangle \equiv\left\langle\left\langle\phi_{\alpha}(t)|H(t)| \phi_{\alpha}(t)\right\rangle\right\rangle .
$$

It is related to the corresponding quasienergy by

$$
E_{\alpha}=\epsilon_{\alpha}+\left\langle\left\langle\phi_{\alpha}(t)\left|\mathrm{i} \hbar \frac{\partial}{\partial t}\right| \phi_{\alpha}(t)\right\rangle\right\rangle
$$

where the outer angle brackets denote the time average over one period of the driving, as indicated in eq. (8). The second term on the right-hand side plays the rôle of a geometric 
phase accumulated over this period [30]. Without the driving, $E_{\alpha}=\epsilon_{\alpha}$, as it should be. By inserting the Fourier expansion (7) the mean energy takes the form

$$
E_{\alpha}=\sum_{n}\left(\epsilon_{\alpha}+n \hbar \omega\right)\left\langle c_{\alpha, n} \mid c_{\alpha, n}\right\rangle
$$

It shows that the $n$th Floquet channel gives a contribution $\epsilon_{\alpha}+n \hbar \omega$ to the mean energy, weighted by the Fourier coefficient $\left\langle c_{\alpha, n} \mid c_{\alpha, n}\right\rangle$ [29].

Quasienergies and Floquet states are obtained numerically by solving the matrix eigenvalue equation 25, 28, 29.

$$
\sum_{n^{\prime}} \sum_{k^{\prime}} H_{n, k ; n^{\prime}, k^{\prime}} c_{n^{\prime}, k^{\prime}}=\epsilon c_{n, k}
$$

equivalent to the time-dependent Schrödinger equation. It is derived by inserting the eigenstates (5) into the Schrödinger equation, Fourier expanding, and using the representation by the eigenstates of the unperturbed Hamiltonian, $H_{0}\left|\Psi_{k}\right\rangle=E_{k}\left|\Psi_{k}\right\rangle$. We introduced the abbreviations

$$
\begin{aligned}
H_{n, k ; n^{\prime}, k^{\prime}}= & \left(E_{k}-n \hbar \omega\right) \delta_{n-n^{\prime}} \delta_{k-k^{\prime}} \\
& +\frac{1}{2} S x_{k, k^{\prime}}\left(\delta_{n-1-n^{\prime}}+\delta_{n+1-n^{\prime}}\right), \\
c_{n, k}= & \left\langle\Psi_{k} \mid c_{n}\right\rangle, \\
x_{k, k^{\prime}}= & \left\langle\Psi_{k}|x| \Psi_{k^{\prime}}\right\rangle .
\end{aligned}
$$

The invariance of the system under $P_{\omega}$ is of considerable help in solving Eq. (11), because it completely decouples the respective systems of eigenvalue equations for the even and odd subspaces [7,8].

\section{B. Coherent chaotic tunneling}

With the driving $H_{1}(x ; t)$ switched off, the classical phase space generated by $H(x, p ; t)$ exhibits the constituent features of a bistable Hamiltonian system. There is a separatrix at $E=0$. It forms the border between two sets of trajectories: One set, with $E<0$, comes in symmetry-related pairs, each partner of which oscillates in either one of the two potential minima. The other set consists of unpaired trajectories, with $E>0$, that encircle both wells in a spatially symmetric fashion.

Increasing the amplitude of the driving from zero onwards has two principal consequences for the classical dynamics (Fig. 2): The separatrix is destroyed as a closed curve and replaced by a homoclinic tangle [31] of stable and unstable manifolds. As a whole, it forms a chaotic layer in the vicinity and with the topology of the former separatrix. It opens the way for diffusive transport between the two potential wells. Due to the nonlinearity of the potential, there is an infinite set of resonances of the driving with the unperturbed motion, both inside and outside the wells [32,33]. Since the period of the unperturbed, closed trajectories diverges for $E \rightarrow 0$, the resonances accumulate towards the separatrix of the unperturbed system. By its sheer phase-space area, the first resonance (the one for which the periods of 
the driving and of the unperturbed oscillation are in a ratio of 1:1) is prominent among the others and soon (in terms of increasing amplitude $F$ ) exceeds the size of the "order-zero" regular areas near the bottom of each well. For the values of $F$ and $\omega$ chosen in the numerical calculations in this paper, all higher resonances remain negligible in size. The borderline between the chaotic layer along the former separatrix and the regular regions within and outside the wells is therefore quite sharply defined. The "coastal strip" formed by hierarchies of regular islands around higher resonances remains narrow. For the tunneling dynamics, the rôle of states located in the border region [11] is therefore not significant here.

Both major tendencies in the evolution of the classical phase space extension of the chaotic layer and growth of the first resonance - leave their specific traces in the quasienergy spectrum. The tunnel doublets characterizing the unperturbed spectrum for $E<0$ pertain to states located on pairs of symmetry-related quantizing tori in the regular regions within the wells. With increasing size of the chaotic layer, the quantizing tori successively resolve in the chaotic sea. The corresponding doublets disappear as distinct structures in the spectrum as they attain a splitting of the same order as the mean level separation. The gradual widening of the doublets proceeds as a smooth function of the driving amplitude [7,8]. This function roughly obeys a power law [34], cf. Fig. 3. As soon as a pair of states is no longer supported by any torus-like manifold, including fractal [35] and vague tori [36], the corresponding eigenvalues detach themselves from the regular ladder to which they formerly belonged. They can then fluctuate freely in the spectrum and thereby "collide" with other chaotic singlets or regular doublets.

The appearance of a regular region, large enough to accomodate several eigenstates, around the first resonance introduces a second ladder of doublets into the spectrum. Size and shape of the first resonance vary in a way different from the fate of the main regular region. The corresponding doublet ladder therefore moves in the spectrum independently of the doublets that pertain to the main regular region, and of the chaotic singlets. This gives rise to additional singlet-doublet and even to doublet-doublet encounters.

\section{Three-level crossings}

Among the various types of quasienergy crossings that occur according to the above scenario, those involving a regular doublet and a chaotic singlet are the most common. In order to give a quantitative account of such crossings and the associated coherent dynamics, and for later reference in the context of the incoherent dynamics, we shall now discuss them in terms of a simple three-state model, devised much in the spirit of Ref. [5].

Far to the left of the crossing, we expect the following situation: There is a doublet of Floquet states

$$
\begin{aligned}
& \left|\psi_{\mathrm{r}}^{+}(t)\right\rangle=\mathrm{e}^{-\mathrm{i} \epsilon_{\mathrm{r}}^{+} t / \hbar}\left|\phi_{\mathrm{r}}^{+}(t)\right\rangle, \\
& \left|\psi_{\mathrm{r}}^{-}(t)\right\rangle=\mathrm{e}^{-\mathrm{i}\left(\epsilon_{\mathrm{r}}^{+}+\Delta\right) t / \hbar}\left|\phi_{\mathrm{r}}^{-}(t)\right\rangle,
\end{aligned}
$$

with even (superscript '+') and odd ('-') generalized parity, respectively, residing on a pair of quantizing tori in one of the regular (subscript ' $r$ ') regions. We have assumed that the quasienergy splitting (as opposed to the unperturbed splitting) is $\epsilon_{\mathrm{r}}^{-}-\epsilon_{\mathrm{r}}^{+}=\Delta>0$. The global relative phases can be chosen such that the superpositions 


$$
\left|\psi_{\mathrm{R}, \mathrm{L}}(t)\right\rangle=\frac{1}{\sqrt{2}}\left(\left|\psi_{\mathrm{r}}^{+}(t)\right\rangle \pm\left|\psi_{\mathrm{r}}^{-}(t)\right\rangle\right)
$$

are initially localized in the right and the left well, respectively, and tunnel back and forth with a frequency $\Delta / \hbar$ given by the tunnel splitting in the presence of the driving.

As the third player, we introduce a Floquet state

$$
\left|\psi_{\mathrm{c}}^{-}(t)\right\rangle=\mathrm{e}^{-\mathrm{i}\left(\epsilon_{\mathrm{r}}^{+}+\Delta+\Delta_{\mathrm{c}}\right) t / \hbar}\left|\phi_{\mathrm{c}}^{-}(t)\right\rangle,
$$

located mainly in the chaotic (subscript 'c') layer, so that its time-periodic part $\left|\phi_{\mathrm{c}}^{-}(t)\right\rangle$ contains a large number of harmonics. Without loss of generality, its generalized parity is fixed to be odd. For the quasienergy, we have assumed that $\epsilon_{\mathrm{c}}^{-}=\epsilon_{\mathrm{r}}^{+}+\Delta+\Delta_{\mathrm{c}}$, where $\left|\Delta_{\mathrm{c}}\right|$ can be regarded as a measure of the distance from the crossing.

The structure of the classical phase space then implies that the mean energy of the chaotic state should be close to the top of the barrier and far above that of the doublet. We assume, like for the quasienergies, a small splitting of the mean energies pertaining to the regular doublet, $E_{\mathrm{r}}^{-}-E_{\mathrm{r}}^{+} \ll E_{\mathrm{c}}^{-}-E_{\mathrm{r}}^{ \pm}$.

In order to model an avoided crossing between $\left|\psi_{\mathrm{r}}^{-}\right\rangle$and $\left|\psi_{\mathrm{c}}^{-}\right\rangle$, we suppose that there is a non-vanishing matrix element $\left\langle\left\langle\phi_{\mathrm{r}}^{-}\left|H_{\mathrm{DW}}\right| \phi_{\mathrm{c}}^{-}\right\rangle\right\rangle=b>0$. For the singlet-doublet crossings under study, we typically find that $\Delta \ll b \ll \hbar \omega$. Neglecting the coupling with all other states, we obtain the three-state (subscript ' $3 \mathrm{~s}$ ') Floquet Hamiltonian

$$
\mathcal{H}_{3 \mathrm{~s}}=\epsilon_{\mathrm{r}}^{+}+\left(\begin{array}{ccc}
0 & 0 & 0 \\
0 & \Delta & b \\
0 & b & \Delta+\Delta_{\mathrm{c}}
\end{array}\right),
$$

in the three-dimensional Hilbert space spanned by $\left\{\left|\phi_{\mathrm{r}}^{+}(t)\right\rangle,\left|\phi_{\mathrm{r}}^{-}(t)\right\rangle,\left|\phi_{\mathrm{c}}^{-}(t)\right\rangle\right\}$. Its Floquet states read

$$
\begin{aligned}
\left|\psi_{0}^{+}(t)\right\rangle & =\mathrm{e}^{-\mathrm{i} \epsilon_{0}^{+} t / \hbar}\left|\phi_{\mathrm{r}}^{+}(t)\right\rangle, \\
\left|\psi_{1}^{-}(t)\right\rangle & =\mathrm{e}^{-\mathrm{i} \epsilon_{1}^{-} t / \hbar}\left(\left|\phi_{\mathrm{r}}^{-}(t)\right\rangle \cos \beta-\left|\phi_{\mathrm{c}}^{-}(t)\right\rangle \sin \beta\right), \\
\left|\psi_{2}^{-}(t)\right\rangle & =\mathrm{e}^{-\mathrm{i} \epsilon_{2}^{-} t / \hbar}\left(\left|\phi_{\mathrm{r}}^{-}(t)\right\rangle \sin \beta+\left|\phi_{\mathrm{c}}^{-}(t)\right\rangle \cos \beta\right) .
\end{aligned}
$$

Their quasienergies are

$$
\epsilon_{0}^{+}=\epsilon_{\mathrm{r}}^{+}, \quad \epsilon_{1,2}^{-}=\epsilon_{\mathrm{r}}^{+}+\Delta+\frac{1}{2} \Delta_{\mathrm{c}} \mp \frac{1}{2} \sqrt{\Delta_{\mathrm{c}}^{2}+4 b^{2}} .
$$

The mean energies are approximately given by

$$
\begin{aligned}
& E_{0}^{+}=E_{\mathrm{r}}^{+}, \\
& E_{1}^{-}=E_{\mathrm{r}}^{-} \cos ^{2} \beta+E_{\mathrm{c}}^{-} \sin ^{2} \beta, \\
& E_{2}^{-}=E_{\mathrm{r}}^{-} \sin ^{2} \beta+E_{\mathrm{c}}^{-} \cos ^{2} \beta,
\end{aligned}
$$

where contributions of the matrix element $b$ have been neglected. The angle $\beta$ describes the mixing between the Floquet states $\left|\psi_{\mathrm{r}}^{-}\right\rangle$and $\left|\psi_{\mathrm{c}}^{-}\right\rangle$and is a measure of the distance to the avoided crossing. By diagonalizing the Hamiltonian (16), we obtain 


$$
2 \beta=\arctan \left(\frac{2 b}{\Delta_{\mathrm{c}}}\right), \quad 0<\beta<\frac{\pi}{2} .
$$

For $\beta \rightarrow \pi / 2$, corresponding to $-\Delta_{\mathrm{c}} \gg b$, we retain the situation far left of the crossing, as outlined above, with $\left|\psi_{1}^{-}\right\rangle \approx\left|\psi_{\mathrm{c}}^{-}\right\rangle,\left|\psi_{2}^{-}\right\rangle \approx\left|\psi_{\mathrm{r}}^{-}\right\rangle$. To the far right of the crossing, i.e., for $\beta \rightarrow 0$ or $\Delta_{\mathrm{c}} \gg b$, the exact eigenstates $\left|\psi_{1}^{-}\right\rangle$and $\left|\psi_{2}^{-}\right\rangle$have interchanged their identity with respect to the phase-space structure [10]. Here, we have $\left|\psi_{1}^{-}\right\rangle \approx\left|\psi_{\mathrm{r}}^{-}\right\rangle$and $\left|\psi_{2}^{-}\right\rangle \approx\left|\psi_{\mathrm{c}}^{-}\right\rangle$. The mean energy is essentially determined by the phase-space structure. Therefore, there is also an exchange of $E_{1}^{-}$and $E_{2}^{-}$in an exact crossing, cf. Eq. (19), while $E_{0}^{+}$remains unaffected (Fig. 4 $4 \mathrm{~b}$ ). The quasienergies $\epsilon_{0}^{+}$and $\epsilon_{1}^{-}$must intersect close to the avoided crossing of $\epsilon_{1}^{-}$ and $\epsilon_{2}^{-}$(Fig. 由a). Their crossing is exact, since they pertain to states with opposite parity (cf. Fig. 1 $1 \mathrm{a}, \mathrm{b})$.

Numerical evidence shows that this idealized picture is not always correct. It may well happen that even far away from a crossing, the doublet splitting does not exactly return to its value on the opposite side (cf. Figs. 5a, 6). It is even possible that an exact crossing of $\epsilon_{0}^{+}$and $\epsilon_{1}^{-}$does not take place at all in the vicinity of the crossing. In that case, the relation of the quasienergies in the doublet gets reversed via the crossing (Fig. 11c,d). Nevertheless, the above scenario captures the essential features.

To study the dynamics of the tunneling process, we focus on the state

$$
|\psi(t)\rangle=\frac{1}{\sqrt{2}}\left(\mathrm{e}^{-\mathrm{i} \epsilon_{0}^{+} t / \hbar}\left|\phi_{0}^{+}(t)\right\rangle+\mathrm{e}^{-\mathrm{i} \epsilon_{1}^{-} t / \hbar}\left|\phi_{1}^{-}(t)\right\rangle \cos \beta+\mathrm{e}^{-\mathrm{i} \epsilon_{2}^{-} t / \hbar}\left|\phi_{2}^{-}(t)\right\rangle \sin \beta\right) .
$$

It is constructed such that at $t=0$, it corresponds to the decomposition of $\left(\left|\psi_{\mathrm{r}}^{+}\right\rangle+\left|\psi_{\mathrm{r}}^{-}\right\rangle\right) / \sqrt{2}$ (cf. Eq. (14)) in the basis (17) at finite distance from the crossing. Therefore, it is initially localized in the regular region in the right well and follows the time evolution under the Hamiltonian (16). From Eqs. (14), (17), we find the probabilities for its evolving into $\left|\psi_{\mathrm{R}}\right\rangle$, $\left|\psi_{\mathrm{L}}\right\rangle$, or $\left|\psi_{\mathrm{c}}\right\rangle$, respectively, to be

$$
\begin{aligned}
P_{\mathrm{R}}(t) & =\left|\left\langle\psi_{\mathrm{R}}(t) \mid \psi(t)\right\rangle\right|^{2} \\
& =\frac{1}{2}\left(1+\cos \frac{\epsilon_{1} t}{\hbar} \cos ^{2} \beta+\cos \frac{\epsilon_{2} t}{\hbar} \sin ^{2} \beta+\left[\cos \frac{\left(\epsilon_{1}-\epsilon_{2}\right) t}{\hbar}-1\right] \cos ^{2} \beta \sin ^{2} \beta\right), \\
P_{\mathrm{L}}(t) & =\left|\left\langle\psi_{\mathrm{L}}(t) \mid \psi(t)\right\rangle\right|^{2} \\
& =\frac{1}{2}\left(1-\cos \frac{\epsilon_{1} t}{\hbar} \cos ^{2} \beta-\cos \frac{\epsilon_{2} t}{\hbar} \sin ^{2} \beta+\left[\cos \frac{\left(\epsilon_{1}-\epsilon_{2}\right) t}{\hbar}-1\right] \cos ^{2} \beta \sin ^{2} \beta\right), \\
P_{\mathrm{c}}(t) & =\left|\left\langle\psi_{\mathrm{c}}(t) \mid \psi(t)\right\rangle\right|^{2}=\left[1-\cos \frac{\left(\epsilon_{1}-\epsilon_{2}\right) t}{\hbar}\right] \cos ^{2} \beta \sin ^{2} \beta .
\end{aligned}
$$

In sufficient distance from the crossing, there is only little mixing between the regular and the chaotic states, i.e., $\sin \beta \ll 1$ or $\cos \beta \ll 1$. The tunneling process then follows the familiar two-state dynamics involving only $\left|\psi_{\mathrm{r}}^{+}\right\rangle$and $\left|\psi_{\mathrm{r}}^{-}\right\rangle$, with tunnel frequency $\Delta / \hbar$ (Fig. 7a).

Close to the avoided crossing, $\cos \beta$ and $\sin \beta$ are of the same order of magnitude, and $\left|\psi_{1}^{-}\right\rangle,\left|\psi_{2}^{-}\right\rangle$become very similar to one another. Both have now support in the chaotic layer as well as in the symmetry-related regular regions and thus are of a hybrid nature. Here, the 
tunneling involves all the three states and must at least be described by a three-level system. The exchange of probability between the two regular regions proceeds via a "stop-over" in the chaotic region [5, [10]. The three quasienergy differences that determine the time scales of this process are in general all different, leading to complicated beats (Fig. 7b).

However, for $\Delta_{\mathrm{c}}=-\Delta / 2$, the two quasienergies $\epsilon_{1}^{-}$and $-\epsilon_{2}^{-}$degenerate. At this point, which marks the center of the crossing, the number of different frequencies in the three-level dynamics reduces to two again. This restores the familiar coherent tunneling in the sense that there is again a simple periodic exchange of probability between the regular regions [10]. However, the rate is much larger if compared to the situation far off the crossing, and the chaotic region is now temporarily populated during each probability transfer, twice per tunneling cycle (Fig. 7]c).

In order to illustrate the above three-state model and to demonstrate its adequacy, we have numerically studied a singlet-doublet crossing that occurs for the double-well potential, Eq. (11), with $D / \hbar=4$, at a driving frequency $\omega=0.982$ and amplitude $F=S / \sqrt{8 D}=$ 0.015029 (Figs. 5, 6). A comparison (not shown) of the appropriately scaled three-state theory (Fig. đ) with this real singlet-doublet crossing (Fig. 5) shows satisfactory agreement. Note that in the real crossing, the quasienergy of the chaotic singlet decreases as a function of $F$, so that the exact crossing occurs to the left of the avoided one.

\section{INCOHERENT QUANTUM DYNAMICS}

\section{A. Master equation}

\section{System-bath model}

To achieve a microscopic model of dissipation, we couple the system (1) bilinearly to a bath of non-interacting harmonic oscillators [37]. The total Hamiltonian of system and bath is then given by

$$
H(t)=H_{\mathrm{DW}}(t)+\sum_{\nu=1}^{\infty}\left(\frac{p_{\nu}^{2}}{2 m_{\nu}}+\frac{m_{\nu}}{2} \omega_{\nu}^{2}\left(x_{\nu}-\frac{g_{\nu}}{m_{\nu} \omega_{\nu}^{2}} x\right)^{2}\right) .
$$

We here couple the position $x$ of the system to an ensemble of oscillators with masses $m_{\nu}$, frequencies $\omega_{\nu}$, momenta $p_{\nu}$, and coordinates $x_{\nu}$, with the coupling strength $g_{\nu}$. The bath is fully characterized by the spectral density of the coupling energy,

$$
J(\omega)=\pi \sum_{\nu=1}^{\infty} \frac{g_{\nu}^{2}}{2 m_{\nu} \omega_{\nu}} \delta\left(\omega-\omega_{\nu}\right)
$$

For the time evolution we choose an initial condition of the Feynman-Vernon type: at $t=t_{0}$, the bath is in thermal equilibrium and uncorrelated to the system, i.e.,

$$
\rho\left(t_{0}\right)=\rho_{\mathrm{S}}\left(t_{0}\right) \otimes \rho_{\mathrm{B}, \mathrm{eq}},
$$

where $\rho_{\mathrm{B}, \mathrm{eq}}=\exp \left(-\beta H_{\mathrm{B}}\right) / \operatorname{tr}_{\mathrm{B}} \exp \left(-\beta H_{\mathrm{B}}\right)$ is the canonical ensemble of the bath and $1 / \beta=$ $k_{\mathrm{B}} T$. 
Due to the bilinearity of the system-bath coupling, one can always eliminate the bath variables to get an exact, closed integro-differential equation for the reduced density matrix

$\rho_{\mathrm{S}}(t)=\operatorname{tr}_{\mathrm{B}} \rho(t)$, which describes the dynamics of the central system, subject to dissipation [38].

\section{Born-Markov approximation}

In most cases, however, the integro-differential equation for $\rho_{\mathrm{S}}(t)$ can be solved only approximately. In particular, in the limit of weak coupling,

$$
\begin{aligned}
& \gamma \ll k_{\mathrm{B}} T / \hbar, \\
& \gamma \ll\left|\epsilon_{\alpha}-\epsilon_{\alpha^{\prime}}\right| / \hbar,
\end{aligned}
$$

it is possible to truncate the time-dependent perturbation expansion in the system-bath interaction after the second-order term. The quantity $\gamma$, to be defined below, denotes the effective damping of the dissipative system, and $\left|\epsilon_{\alpha}-\epsilon_{\alpha^{\prime}}\right| / \hbar$ are the transition frequencies of the central system. In the present case, the central system is understood to include the driving [20 23], so that the transition frequencies are given by quasienergy differences. The autocorrelations of the bath decay on a time scale $\hbar / k_{\mathrm{B}} T$ and thus in the present limit, instantaneously on the time scale $1 / \gamma$ of the system correlations. With the initial preparation (25), the equation of motion for the reduced density matrix in this approximation is given by [23]

$$
\begin{aligned}
\dot{\rho}_{\mathrm{S}}(t)= & -\frac{\mathrm{i}}{\hbar}\left[H_{\mathrm{S}}(t), \rho_{\mathrm{S}}(t)\right]+\frac{1}{\pi \hbar} \int_{-\infty}^{\infty} \mathrm{d} \omega J(\omega) n_{\mathrm{th}}(\hbar \omega) \\
& \times \int_{0}^{\infty} \mathrm{d} \tau\left(\mathrm{e}^{\mathrm{i} \omega \tau}\left[\tilde{x}(t-\tau, t) \rho_{\mathrm{S}}(t), x\right]+\text { H.c. }\right)
\end{aligned}
$$

where $\tilde{x}\left(t^{\prime}, t\right)$ denotes the position operator in the interaction picture defined by

$$
\tilde{x}\left(t^{\prime}, t\right)=U^{\dagger}\left(t^{\prime}, t\right) x U\left(t^{\prime}, t\right),
$$

with $U\left(t^{\prime}, t\right)$, the propagator of the conservative driven double well, given in Eq. (6). 'H.c.' denotes the Hermitian conjugate and

$$
n_{\mathrm{th}}(\epsilon)=\frac{1}{\mathrm{e}^{\epsilon / k_{\mathrm{B}} T}-1}=-n_{\mathrm{th}}(-\epsilon)-1
$$

is the thermal occupation of the bath oscillator with energy $\epsilon$. To achieve a more compact notation, we require $J(-\omega)=-J(\omega)$. In the following, we shall restrict ourselves to an Ohmic bath, $J(\omega)=m \gamma \omega$. This defines the effective damping constant $\gamma$.

We use the time-periodic components $\left|\phi_{\alpha}(t)\right\rangle$ of the Floquet states as a basis to expand the density operator, Eq. (28). Expressing the matrix elements

$$
X_{\alpha \beta}(t)=\left\langle\phi_{\alpha}(t)|x| \phi_{\beta}(t)\right\rangle
$$

of the position operator by their Fourier coefficients 


$$
\begin{aligned}
X_{\alpha \beta, n} & =\left\langle\left\langle\phi_{\alpha}(t)\left|x \mathrm{e}^{-\mathrm{i} n \omega t}\right| \phi_{\beta}(t)\right\rangle\right\rangle=X_{\beta \alpha,-n}^{*}, \\
X_{\alpha \beta}(t) & =\sum_{n} \mathrm{e}^{\mathrm{i} n \omega t} X_{\alpha \beta, n},
\end{aligned}
$$

yields for the matrix elements $\sigma_{\alpha \beta}$ of the reduced density matrix $\rho_{\mathrm{S}}$ the equation of motion 18, 19,21,23

$$
\begin{aligned}
\dot{\sigma}_{\alpha \beta}(t)= & \frac{\mathrm{d}}{\mathrm{d} t}\left\langle\phi_{\alpha}(t)\left|\rho_{\mathrm{S}}(t)\right| \phi_{\beta}(t)\right\rangle \\
= & -\frac{\mathrm{i}}{\hbar}\left(\epsilon_{\alpha}-\epsilon_{\beta}\right) \sigma_{\alpha \beta}(t) \\
& +\sum_{\alpha^{\prime} \beta^{\prime} n n^{\prime}}\left(N_{\alpha \alpha^{\prime}, n} X_{\alpha \alpha^{\prime}, n} \sigma_{\alpha^{\prime} \beta^{\prime}} X_{\beta^{\prime} \beta, n^{\prime}}\right. \\
& \left.-N_{\alpha^{\prime} \beta^{\prime}, n} X_{\alpha \alpha^{\prime}, n^{\prime}} X_{\alpha \alpha^{\prime}, n} \sigma_{\beta^{\prime} \beta}\right) \mathrm{e}^{\mathrm{i}\left(n+n^{\prime}\right) \omega t}+\text { H.c. }
\end{aligned}
$$

Note that the coefficients of this differential equation are periodic in time with the period of the driving. The $N_{\alpha \beta, n}$ are given by

$$
N_{\alpha \beta, n}=N\left(\epsilon_{\alpha}-\epsilon_{\beta}+n \hbar \omega\right), \quad N(\epsilon)=\frac{m \gamma \epsilon}{\hbar^{2}} n_{\mathrm{th}}(\epsilon) .
$$

For $\epsilon \gg k_{\mathrm{B}} T, N(\epsilon)$ approaches zero.

Since the position operator $x$ is odd under transformation with the generalized parity (3), the master equations (28) and (34) are invariant under transformation with $\mathrm{P}_{\omega}$. Therefore, both the conservative and the dissipative dynamics preserve the parity of the operator $\left|\phi_{\alpha}\right\rangle\left\langle\phi_{\beta}\right|$. It is even if $\left|\phi_{\alpha}\right\rangle$ and $\left|\phi_{\beta}\right\rangle$ belong to the same parity class and odd otherwise. Note that in particular, the projectors $\left|\phi_{\alpha}\right\rangle\left\langle\phi_{\alpha}\right|$ and thus all density matrices diagonal in the Floquet basis are even under $\mathrm{P}_{\omega}$.

\section{Rotating-wave approximation}

Assuming that dissipative effects are relevant only on a time scale much larger than the period $2 \pi / \omega$ of the driving, we average the coefficients of the master equation (34) over $2 \pi / \omega$ and end up with the equation of motion

$$
\dot{\sigma}_{\alpha \beta}(t)=-\frac{\mathrm{i}}{\hbar}\left(\epsilon_{\alpha}-\epsilon_{\beta}\right) \sigma_{\alpha \beta}(t)+\sum_{\alpha^{\prime} \beta^{\prime}} L_{\alpha \beta \alpha^{\prime} \beta^{\prime}} \sigma_{\alpha^{\prime} \beta^{\prime}},
$$

with the time-independent dissipative part

$$
\begin{aligned}
L_{\alpha \beta \alpha^{\prime} \beta^{\prime}}= & \sum_{n}\left(N_{\alpha \alpha^{\prime}, n}+N_{\beta \beta^{\prime}, n}\right) X_{\alpha \alpha^{\prime}, n} X_{\beta^{\prime} \beta,-n} \\
& -\delta_{\beta \beta^{\prime}} \sum_{\beta^{\prime \prime}, n} N_{\beta^{\prime \prime} \alpha^{\prime}, n} X_{\alpha \beta^{\prime \prime},-n} X_{\beta^{\prime \prime} \alpha^{\prime}, n} \\
& -\delta_{\alpha \alpha^{\prime}} \sum_{\alpha^{\prime \prime} n} N_{\alpha^{\prime \prime} \beta^{\prime}, n} X_{\beta^{\prime} \alpha^{\prime \prime},-n} X_{\alpha^{\prime \prime} \beta, n} .
\end{aligned}
$$

This step amounts effectively to a rotating-wave approximation. It is, however, less restrictive than the rotating-wave approximation introduced in [20,21] where dissipative effects are averaged over the generally longer time scale $\max _{\alpha, \beta, n}\left(2 \pi \hbar /\left(\epsilon_{\alpha}-\epsilon_{\beta}+n \hbar \omega\right)\right)$. 


\section{B. Dissipative chaos-assisted tunneling}

The crucial effect of dissipation on a quantum system is the disruption of coherence: a coherent superposition evolves into an incoherent mixture. Thus, phenomena based on coherence, such as tunneling, are rendered transients that fade out on a finite time scale $t_{\text {decoh }}$. In general, for driven tunneling in the weakly damped regime, this time scale gets shorter for higher temperatures, reflecting the growth of transition rates [39. However, there exist counterintuitive effects. For example, in the vicinity of an exact crossing of the ground-state quasienergies, the coherent suppression of tunneling [15] can be stabilized with higher temperatures [18, [1, 40] until levels outside the doublet start to play a rôle. We have studied dissipative chaos-assisted tunneling numerically, using again the real singlet-doublet crossing introduced in Sect. П10 (cf. Fig. 5) as our working example.

In the vicinity of a singlet-doublet crossing, the tunnel splitting increases significantlythe essence of chaos-assisted tunneling. During the tunneling, the chaotic singlet becomes populated periodically with frequency $\left|\epsilon_{2}^{-}-\epsilon_{1}^{-}\right| / \hbar$, cf. Eq. (22) and Fig. 1. The high mean energy of this singlet results in an enhanced decay of coherence at times when $\left|\psi_{\mathrm{c}}\right\rangle$ is populated (Fig. 8). For the relaxation towards the asymptotic state, also the slower transitions within doublets are relevant. Therefore, the corresponding time scale $t_{\text {relax }}$ can be much larger than $t_{\text {decoh }}$ (Fig. 9).

To obtain quantitative estimates for the dissipative time scales, we approximate $t_{\text {decoh }}$ by the decay rate of $\operatorname{tr} \rho^{2}$, a measure of coherence, averaged over time,

$$
\begin{aligned}
\frac{1}{t_{\text {decoh }}} & =-\frac{1}{t} \int_{0}^{t} \mathrm{~d} t^{\prime} \frac{\mathrm{d}}{\mathrm{d} t^{\prime}} \operatorname{tr} \rho^{2}\left(t^{\prime}\right) \\
& =\frac{1}{t}\left(\operatorname{tr} \rho^{2}(0)-\operatorname{tr} \rho^{2}(t)\right) .
\end{aligned}
$$

Because of the stepwise decay of the coherence (Fig. 8), we have chosen the propagation time $t$ as an $n$-fold multiple of the duration $2 \pi /\left|\epsilon_{2}^{-}-\epsilon_{1}^{-}\right|$of the chaotic beats. For this procedure to be meaningful, $n$ should be so large that the coherence decays substantially during the time $t$ (in our numerical studies to a value of approximatly 0.9). The time scale $t_{\text {relax }}$ of the approach to the asymptotic state is given by the reciprocal of the smallest real part of the eigenvalues of the dissipative kernel.

Outside the singlet-doublet crossing we find that the decay of coherence and the relaxation take place on roughly the same time scale (Fig. 10). At $F \approx 0.013$, the chaotic singlet induces an exact crossing of the ground-state quasienergies (see Fig. 6), resulting in a stabilization of coherence with increasing temperature. At the center of the avoided crossing, the decay of coherence becomes much faster and is essentially independent of temperature. This indicates that transitions from states with mean energy far above the ground state play a crucial rôle.

\section{Asymptotic state}

As the dynamics described by the master equation (28) is dissipative, it converges in the long-time limit to an asymptotic state $\rho_{\infty}(t)$. In general, this attractor remains time 
dependent but shares all the symmetries of the central system, i.e. here, periodicity and generalized parity. However, the coefficients of the master equation (37) for the matrix elements $\sigma_{\alpha \beta}$, valid within a rotating-wave approximation, are time independent and so the asymptotic solution also is. This means that we have eliminated the explicit time dependence of the attractor by representing it in the Floquet basis and introducing a mild rotating-wave approximation.

To gain some qualitative insight into the asymptotic solution, we focus on the diagonal elements

$$
L_{\alpha \alpha \alpha^{\prime} \alpha^{\prime}}=2 \sum_{n} N_{\alpha \alpha^{\prime}, n}\left|X_{\alpha \alpha^{\prime}, n}\right|^{2}, \quad \alpha \neq \alpha^{\prime},
$$

of the dissipative kernel. They give the rates of the direct transitions from $\left|\phi_{\alpha^{\prime}}\right\rangle$ to $\left|\phi_{\alpha}\right\rangle$. Within a cruder rotating-wave approximation [21], these are the only non-vanishing contributions to the master equation which affect the diagonal elements $\sigma_{\alpha \alpha}$ of the density matrix.

In the case of zero driving amplitude, the Floquet states $\left|\phi_{\alpha}\right\rangle$ reduce to the eigenstates of the undriven Hamiltonian $H_{\mathrm{DW}}$. The only non-vanishing Fourier component is then $\left|c_{\alpha, 0}\right\rangle$, and the quasienergies $\epsilon_{\alpha}$ reduce to the corresponding eigenenergies $E_{\alpha}$. Thus $L_{\alpha \alpha \alpha^{\prime} \alpha^{\prime}}$ consists of a single term proportional to $N\left(\epsilon_{\alpha}-\epsilon_{\alpha^{\prime}}\right)$ only. It describes two kinds of thermal transitions: decay to states with lower energy and, if the energy difference is less than $k_{\mathrm{B}} T$, thermal activation to states with higher energy. The ratio of the direct transitions forth and back then reads

$$
\frac{L_{\alpha \alpha \alpha^{\prime} \alpha^{\prime}}}{L_{\alpha^{\prime} \alpha^{\prime} \alpha \alpha}}=\exp \left(-\frac{\left(\epsilon_{\alpha}-\epsilon_{\alpha^{\prime}}\right)}{k_{\mathrm{B}} T}\right)
$$

We have detailed balance. Therefore, the steady-state solution $\sigma_{\alpha \alpha^{\prime}}(\infty) \sim$ $\exp \left(-\epsilon_{\alpha} / k_{\mathrm{B}} T\right) \delta_{\alpha \alpha^{\prime}}$. In particular, the occupation probability decays monotonically with the energy of the eigenstates. In the limit $k_{\mathrm{B}} T \rightarrow 0$, the system tends to occupy the ground state only.

For a strong driving, each Floquet state $\left|\phi_{\alpha}\right\rangle$ contains a large number of Fourier components and $L_{\alpha \alpha \alpha^{\prime} \alpha^{\prime}}$ is given by a sum over contributions with quasienergies $\epsilon_{\alpha}-\epsilon_{\alpha^{\prime}}+n \hbar \omega$. Thus a decay to states with "higher" quasienergy (recall that quasienergies do not allow for a global ordering) becomes possible due to terms with $n<0$. Physically, they describe dissipative transitions under absorption of driving-field quanta. Correspondingly, the system tends to occupy Floquet states comprising many Fourier components with low index $n$. According to Eq. (10), these states have low mean energy.

The effects under study are found for a driving with a frequency of the order of unity. Thus for a quasienergy doublet, i.e., far off the three-level crossing, we have $\left|\epsilon_{\alpha}-\epsilon_{\alpha^{\prime}}\right| \ll \hbar \omega$, and $L_{\alpha^{\prime} \alpha^{\prime} \alpha \alpha}$ is dominated by contributions with $n<0$, where the splitting has only small influence. However, as a consequence of symmetry, the splitting is the main difference between the two partners of the quasienergy doublet. Therefore, with respect to dissipation, both should behave similarly. In particular, one expects an equal population of the doublets even in the limit of zero temperature (Fig. 11a). This is in contrast to the undriven case.

In the vicinity of a singlet-doublet crossing the situation is more subtle. Here, the odd partner, say, of the doublet mixes with a chaotic singlet, cf. Eq. (17), and thus acquires 
components with higher energy. Due to the high mean energy $E_{\mathrm{c}}^{-}$of the chaotic singlet, close to the top of the barrier, the decay back to the ground state can also proceed indirectly via other states with mean energy below $E_{\mathrm{c}}^{-}$. Thus $\left|\phi_{1}^{-}\right\rangle$and $\left|\phi_{2}^{-}\right\rangle$are depleted and mainly $\left|\phi_{0}^{+}\right\rangle$will be populated. However, if the temperature is significantly above the splitting of the avoided crossing, thermal activation from $\left|\phi_{0}^{+}\right\rangle$to $\left|\phi_{1,2}^{-}\right\rangle$, accompanied by depletion via the states below $E_{\mathrm{c}}^{-}$, becomes possible. Thus asymptotically, all these states become populated in a steady flow (Figs. 11]b,c).

An important global characteristic of the asymptotic state is its coherence $\operatorname{tr} \rho_{\infty}^{2}$. Its value gives approximately the reciprocal of the number of incoherently occupied states. It equals unity only if the attractor is a pure state. According to the above scenario, we expect $\operatorname{tr} \rho_{\infty}^{2}$ to assume the value $1 / 2$, in a regime with strong driving but preserved doublet structure, reflecting the incoherent population of the ground-state doublet. In the vicinity of the singlet-doublet crossing where the doublet structure is dissolved, its value should be close to unity for temperatures $k_{\mathrm{B}} T \ll b$ and much less than unity for $k_{\mathrm{B}} T \gg b$ (Figs. 12, 13). This means that the crossing of the chaotic singlet with the regular doublet leads to an improvement of coherence if the temperature is below the splitting of the avoided crossing, and a loss of coherence for temperatures above the splitting. This phenomenon amounts to a chaos-induced coherence or incoherence, respectively.

The crucial rôle of the decay via states not involved in the three-level crossing can be demonstrated by comparing it with the dissipative dynamics including only these three levels (plus the bath). At the crossing, the three-state model results in a completely different type of asymptotic state (Fig. 13). The failure of the three-state model in the presence of dissipation clearly indicates that in the vicinity of the singlet-doublet crossing, it is important to take a large set of levels into account.

\section{CONCLUSION}

Nonlinear systems with a mixed phase space still represent a formidable challenge for a theoretical understanding. On the classical level, the intricate fractal interweaving of regular and chaotic regions of phase space is far from being exhaustively studied. Quantummechanical uncertainty reduces the richness of the classical phase-space structure, but at the same time, coherence effects like tunneling add new elements to the dynamics. For example, in a bistable system, a driving with an energy in the regime between the unperturbed tunnel splittings and the typical separation of doublets leads to transport phenomena that combine tunneling and classical chaotic diffusion as essential elements. Yet another basic energy scale enters if the unavoidable coupling of the system to its environment is taken into account. If the finite width the levels attain in the presence of dissipation is comparable, in turn, to the energies characterizing driven tunneling, unfamiliar phenomena due to the interplay of chaos, tunneling, and decoherence occur in the transient dynamics and possibly also in the asymptotic state of the driven damped quantum system.

In this paper, we have selected a specific case out of the host of problems to be studied in this field: dissipative chaotic tunneling in the vicinity of crossings of chaotic singlets with tunnel doublets. In order to obtain a firm quantitative basis, we have endowed a prototypical model for chaotic tunneling, a harmonically driven double well, with dissipation. Thereby, 
we have followed the usual approach of coupling to a heat bath, but adapted to the periodic time dependence of the central system. Moreover, we have carefully avoided to destroy, by the approximations introduced in the derivation of a master equation, the specific spectral characteristics of chaotic tunneling that we are interested in. As a simple intuitive model to compare against, we have constructed a three-state system which in the case of vanishing dissipation, provides a faithful description of an isolated singlet-doublet crossing.

As an example for the interplay of chaotic tunneling with dissipation, we mention an effect that might be termed "chaos-induced coherence" for short. Suppose that damping and temperature are such that far off the singlet-doublet crossing, the level width is of the same order or larger than the tunnel splitting. There, coherent tunneling is then largely suppressed even as a transient. Close to the crossing, however, the widening of the doublet enforced by the intersecting third level creates a separation of the time scales of the coherent tunneling and of its decay due to incoherent processes, without altering the dissipation as such. As a consequence, the transient dynamics now exhibits many tunneling cycles, each of which includes two "stop overs" in the chaotic phase-space region, one on the way to, one on the way back.

The study of the asymptotic state, the "quantum attractor", demonstrates clearly that a three-state model of the singlet-doublet crossing is no longer adequate once dissipation is effective. This is so because the coupling to the heat bath enables processes of decay and thermal activation that connect the states in the crossing with other, "external" states of the central system. In the presence of driving, the asymptotic state is no longer literally a state of equilibrium. Rather, incoherent processes create a steady flow of probability involving states within as well as outside the crossing. As a result, the composition of the asymptotic state, expressed for example by its coherence $\operatorname{tr} \rho_{\infty}^{2}$, will be markedly different at the center of the crossing as compared to the asymptotic state far away from the crossing, even if that is barely visible in the corresponding phase-space structure.

Many more phenomena at the overlap of chaos, tunneling, and dissipation await being unraveled. They include four-state crossings formed when two doublets intersect, chaotic Bloch tunneling along extended potentials with a large number of unit cells instead of just two, the influence of decoherence on a multi-step mechanism of chaotic tunneling [11.12] and transient tunneling between coexisting strange attractors, to mention only a few. At the same time, analytical tools more specifically taylored to the investigation of the quantumclassical correspondence in mixed systems, such as a phase-space entropy [41, will provide additional insight.

\section{ACKNOWLEDGMENTS}

Financial support of this work by the Deutsche Forschungsgemeinschaft (grant No. Di 511/2-2) is gratefully acknowledged. One of us (TD) would like to thank for the warm hospitality enjoyed during a stay in the group of Prof. O. Bohigas, Institut de Physique Nucléaire, Orsay, financed by Deutsche Forschungsgemeinschaft (grant No. Di 511/4-1), where this work was completed. 


\section{REFERENCES}

[1] M. J. Davis and E. J. Heller, J. Chem. Phys. 75, 246 (1986).

[2] O. Bohigas, S. Tomsovic, and D. Ullmo, Phys. Rev. Lett. 64, 1479 (1990); Phys. Rev. Lett. 65, 5 (1990).

[3] W. A. Lin and L. E. Ballentine, Phys. Rev. Lett. 65, 2927 (1990); Phys. Rev. A 45, 3637 (1992).

[4] J. Plata and J. M. Gomez Llorente, J. Phys. A 25, L303 (1992).

[5] O. Bohigas, S. Tomsovic, and D. Ullmo, Phys. Rep. 223, 43 (1993).

[6] S. Tomsovic and D. Ullmo, Phys. Rev. E 50, 145 (1994).

[7] R. Utermann, T. Dittrich, and P. Hänggi, Physica B 194-196, 1013 (1994); Phys. Rev. E 49, 273 (1994).

[8] R. Utermann, Dissertation, Universität Augsburg, unpublished (1998).

[9] R. Roncaglia, L. Bonci, F. M. Izrailev, B. J. West, and P. Grigolini, Phys. Rev. Lett. 73, 802 (1994).

[10] M. Latka, P. Grigolini, and B. J. West, Phys. Rev. E 50, 596 (1994); Phys. Rev. A 50, 1071 (1994); Phys. Rev. E 50, R3299 (1994).

[11] E. Doron and S. D. Frischat, Phys. Rev. Lett. 75, 3661 (1995).

[12] S. D. Frischat and E. Doron, Phys. Rev. E 57, 1421 (1998).

[13] E. M. Zanardi, J. Gutiérrez, and J. M. Gomez Llorente, Phys. Rev. E 52, 4736 (1995).

[14] F. Leyvraz and D. Ullmo, J. Phys. A 10, 2529 (1996).

[15] F. Grossmann, P. Jung, T. Dittrich, and P. Hänggi, Z. Phys. B 84, 315 (1991); Phys. Rev. Lett. 67, 516 (1991); for a recent review see: M. Grifoni and P. Hänggi, Phys. Rep. (in press).

[16] F. Grossmann, T. Dittrich, P. Jung, and P. Hänggi, J. Stat. Phys. 70, 229 (1993).

[17] F. Grossmann, T. Dittrich, and P. Hänggi, Physica B 175, 293 (1991).

[18] T. Dittrich, B. Oelschlägel, and P. Hänggi, Europhys. Lett. 22, 5 (1993).

[19] T. Dittrich, P. Hänggi, B. Oelschlägel, and R. Utermann, in 25 Years of Non-Equilibrium Statistical Mechanics, J. J. Brey et al. (eds.), Lecture Notes in Physics, Vol. 445, Springer (Berlin, 1995), p. 269.

[20] R. Blümel, R. Graham, L. Sirko, U. Smilansky, H. Walther, and K. Yamada, Phys. Rev. Lett. 62, 341 (1989).

[21] R. Blümel, A. Buchleitner, R. Graham, L. Sirko, U. Smilansky, and H. Walther, Phys. Rev. A 44, 4521 (1991).

[22] R. Graham and R. Hübner, Ann. Phys. (NY) 234, 300 (1994).

[23] S. Kohler, T. Dittrich, and P. Hänggi, Phys. Rev. E 55, 300 (1997).

[24] A. Peres, Phys. Rev. Lett. 67, 158 (1991).

[25] J. H. Shirley, Phys. Rev. 138B, 979 (1965).

[26] H. Sambe, Phys. Rev. A 7, 2203 (1973).

[27] N. L. Manakov, V. D. Ovsiannikov, and L. P. Rapoport, Phys. Rep. 141, 319 (1986).

[28] S. Chu, Adv. Chem. Phys. 73, 739 (1989).

[29] P. Hänggi, in Quantum Transport and Dissipation, T. Dittrich et al., Wiley-VCH (Weinheim, 1998), p. 249.

[30] D. J. Moore, Helv. Phys. Acta 66, 3 (1993). 
[31] A. J. Lichtenberg and M. A. Lieberman, Regular and Stochastic Motion, vol. 38 of Appl. Math. Sci., Springer (New York, 1983).

[32] D. F. Escande, Phys. Rep. 121, 165 (1985).

[33] L. E. Reichl and W. M. Zheng, in Directions in Chaos, vol. 1, H. B. Lin (ed.), World Scientific (Singapore, 1987), p. 17.

[34] M. Wilkinson, Physica 21D, 341 (1986); J. Phys. A 20, 635 (1987).

[35] L. E. Reichl, in The Transition to Chaos: In Conservative and Classical Systems: Quantum Manifestations, Springer (New York, 1992), Chaps. 3.9, 9.5.1, and refs. therein.

[36] W. P. Reinhardt, J. Phys. Chem. 86, 2158 (1982); R. B. Shirts and W. P. Reinhardt, J. Chem. Phys. 77, 5204 (1982).

[37] V. B. Magalinskiı̌, Zh. Eksp. Teor. Fiz. 36, 1942 (1959) [Sov. Phys. JETP 9, 1381 (1959)]; R. Zwanzig, J. Stat. Phys. 9, 215 (1973).

[38] F. Haake, in Quantum Statistics in Optics and Solid-State Physics, Vol. 66 of Springer Tracts in Modern Physics, edited by G. Höhler, Springer (Berlin, 1973), p. 105.

[39] P. Hänggi, P. Talkner, and M. Borkovec, Rev. Mod. Phys. 62, 251 (1990).

[40] D. E. Makarov and N. Makri, Phys. Rev. E 52, 5863 (1995); N. Makri, J. Chem. Phys. 106, 22861 (1997).

[41] B. Mirbach and H. J. Korsch, Phys. Rev. Lett. 75, 362 (1995). 


\section{FIGURES}

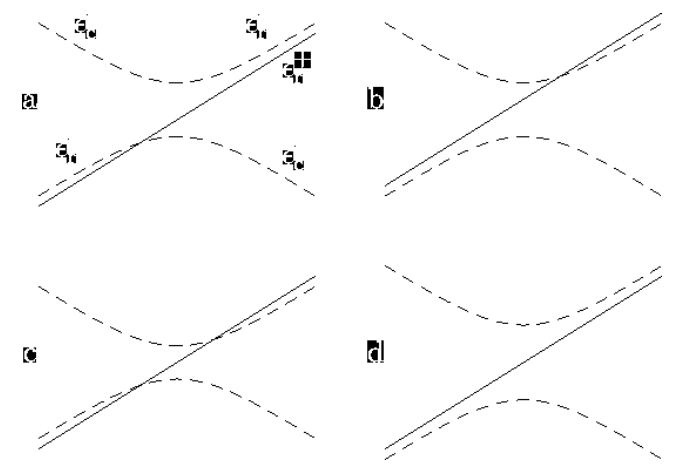

FIG. 1. Possible configurations of quasienergy crossings between a chaotic singlet and a regular doublet. Different line types signify different parity. See Sect. IIC for the labeling of the levels. Note that only for configurations a,b, the order of the regular doublet is restored in passing through the crossing. In configurations c,d, it is reversed.

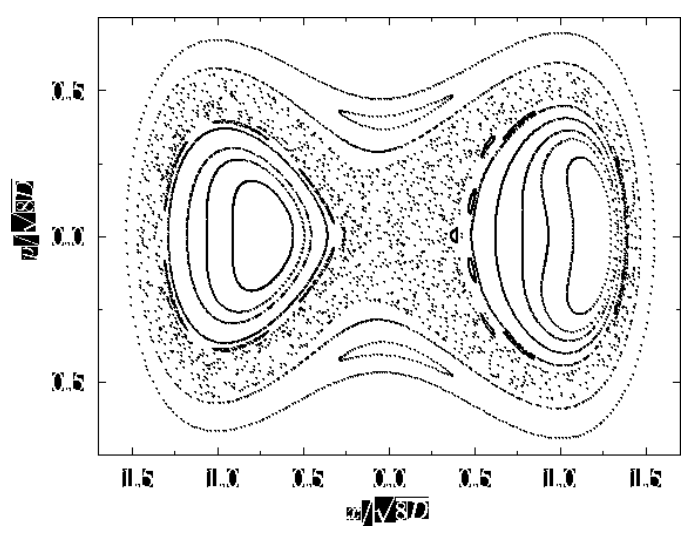

FIG. 2. Stroboscopic classical phase-space portraits, at $\omega t=2 \pi n$, of the harmonically driven quartic double well, Eq. (1), at $F=0.015, \omega=0.982$.

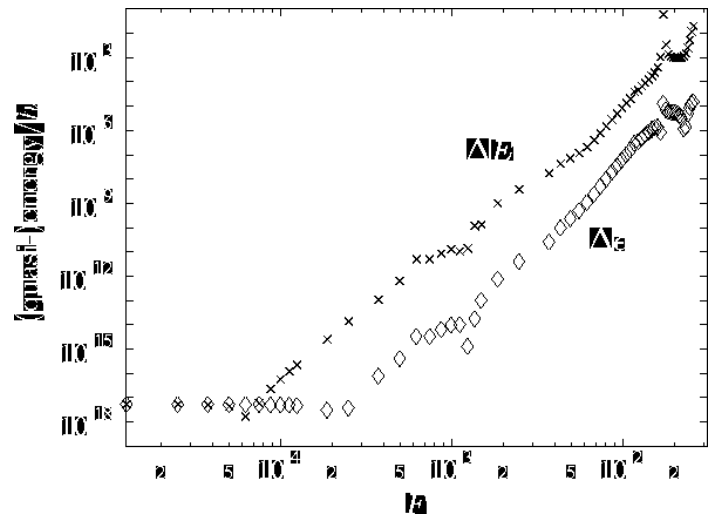


FIG. 3. Dependence on the driving amplitude of the splitting of the ground-state doublet, of the harmonically driven quartic double well, Eq. (11), at $D / \hbar=8$ and $\omega=0.95$. Diamonds are quasienergy, crosses mean-energy splittings. The dips interrupting the smooth parameter dependence indicate crossings of the ground-state doublet with chaotic singlets. After [8].
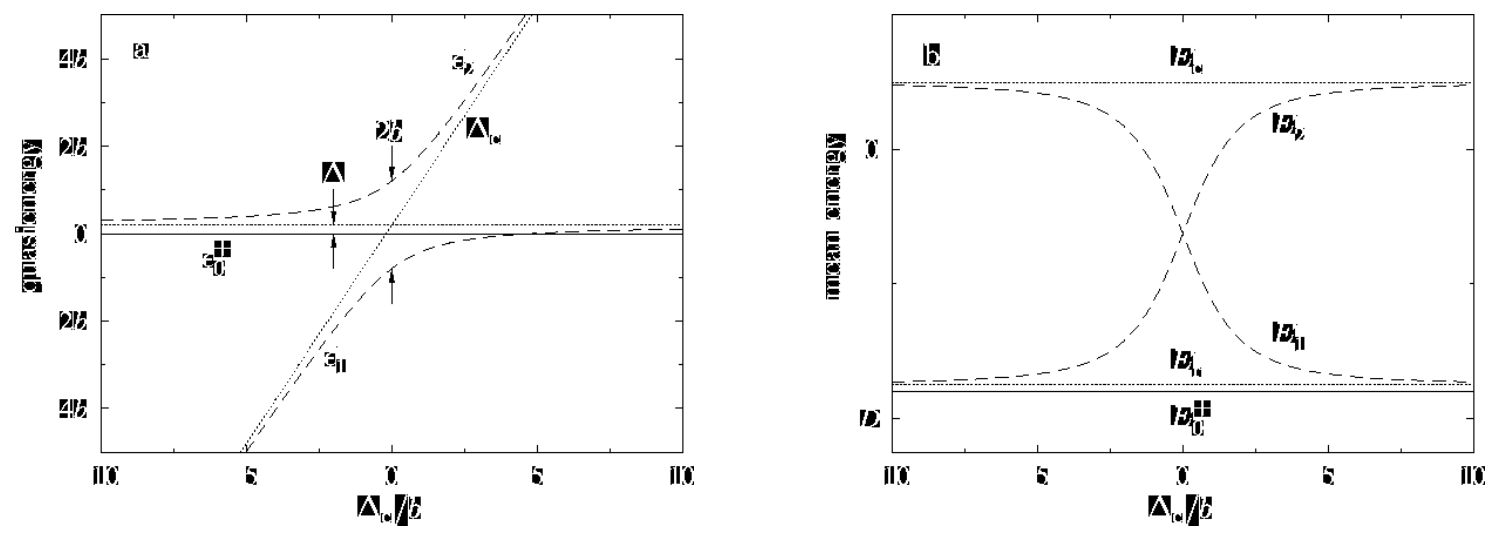

FIG. 4. A singlet-doublet crossing, according to a three-state model, Eq. (16), in terms of the dependence of the quasienergies (panel a) and the mean energies (b) on the coupling parameter $\Delta_{c} / b$. Unperturbed energies are marked by dotted lines, the energies for the case with coupling by full lines for even and dashed lines for odd states.
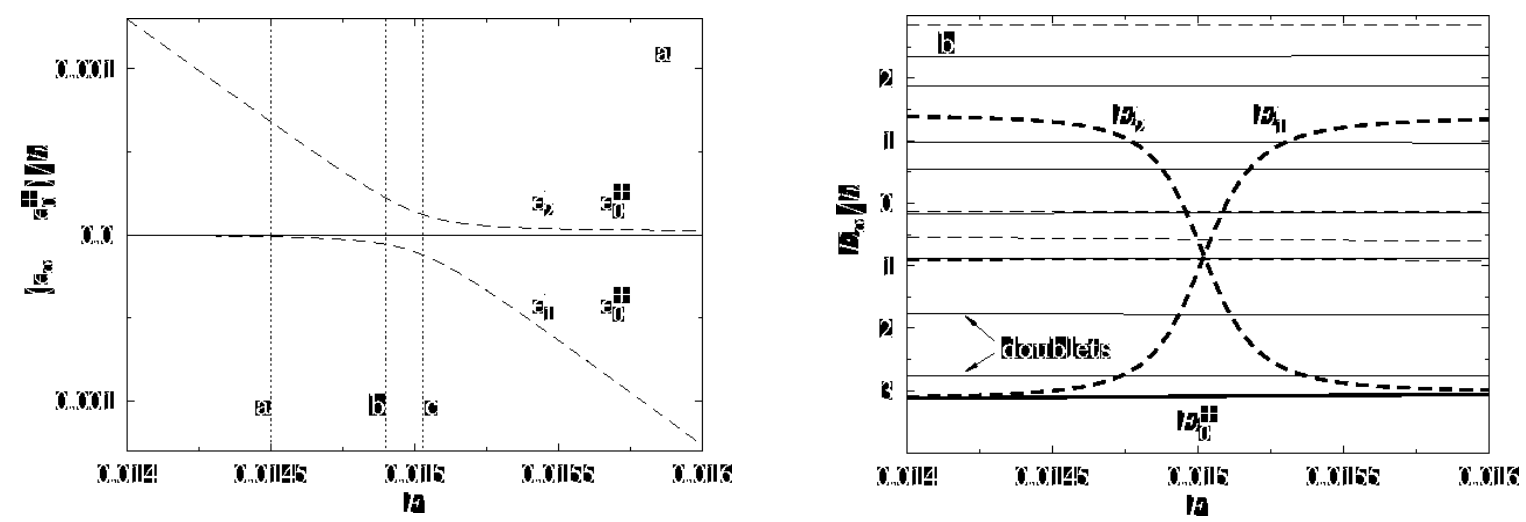

FIG. 5. Singlet-doublet crossing found numerically for the driven double well, Eq. (11), at $D / \hbar=4$ and $\omega=0.982$, in terms of the dependence of the quasienergies (a) and the mean energies (b) on the driving amplitude $F=S / \sqrt{8 D}$. Values of the driving amplitude used in Figs. 目 are marked by dotted vertical lines. Full and dashed lines indicate energies of even and odd states, respectively. Bold lines give the mean energies of the chaotic singlet and the ground-state doublet depicted in panel a. 


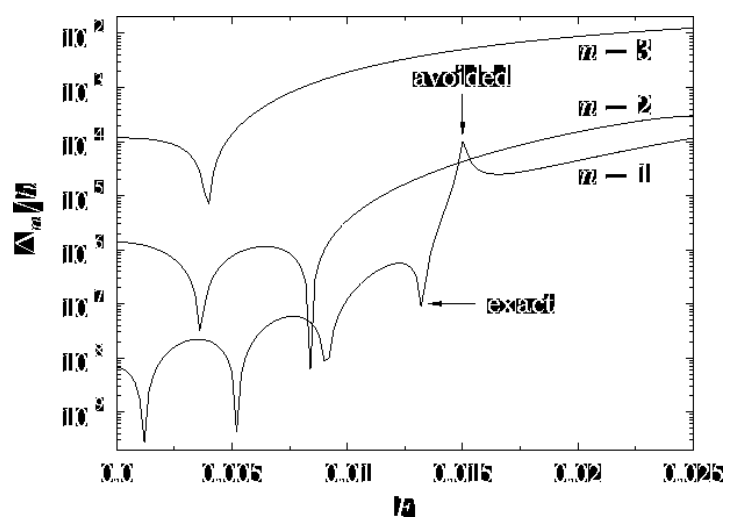

FIG. 6. Splitting of the lowest doublets for $D / \hbar=4$ and $\omega=0.982$. The arrows indicate the locations of the exact and the avoided crossing within a three-level crossing of the type sketched in Fig. 11a.
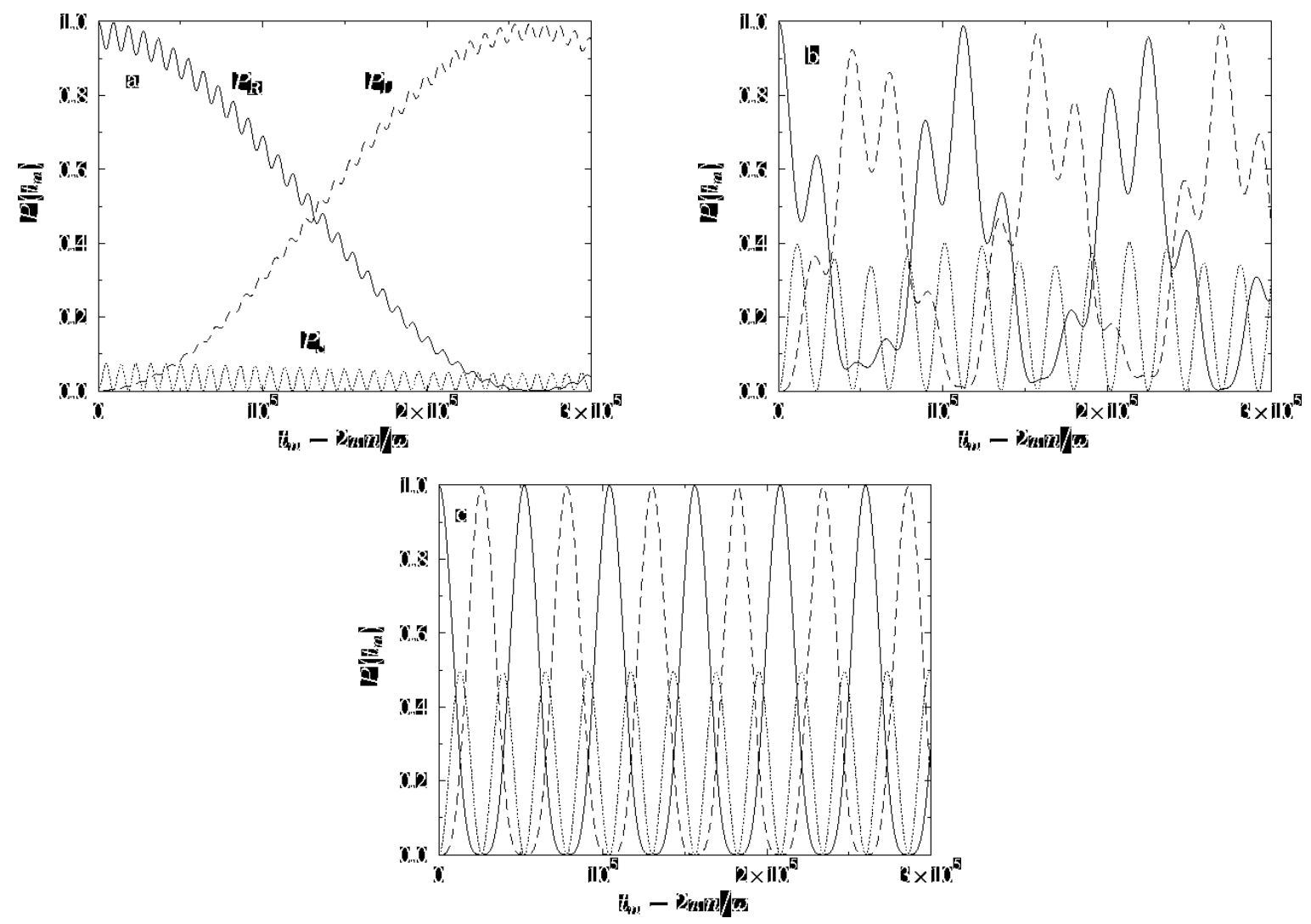

FIG. 7. Time evolution of a state initially localized in the right well, in the vicinity of the singlet-doublet crossing shown in Fig. 5 , in terms of the probabilities to be in the right well (return probability, marked by full lines), in the reflected state in the left well (dashed), or in the chaotic state $\left|\psi_{\mathrm{c}}\right\rangle$ (dotted). Parameter values are as in Fig. 5, and $F=0.0145$ (a), 0.0149 (b), 0.015029 (c). 

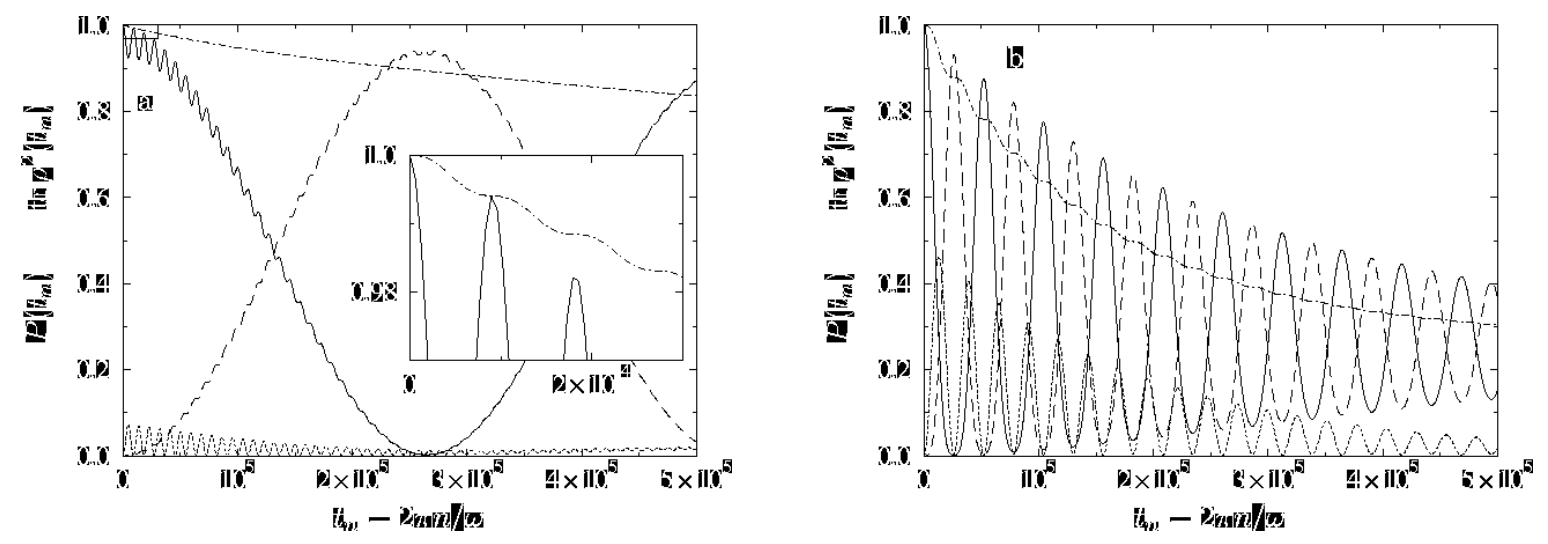

FIG. 8. Occupation probabilities as in Fig. $7 \mathrm{a}, \mathrm{c}$, but in the presence of dissipation. The dash-dotted line shows the time evolution of $\operatorname{tr} \rho^{2}$. The parameter values are $D / \hbar=4, \omega=0.982$, $\gamma=10^{-6}, k_{\mathrm{B}} T / \hbar=10^{-4}$, and $F=0.0145$ (a), 0.015029 (b). The inset in (a) is a blow up of the rectangle in the upper left corner of that panel.

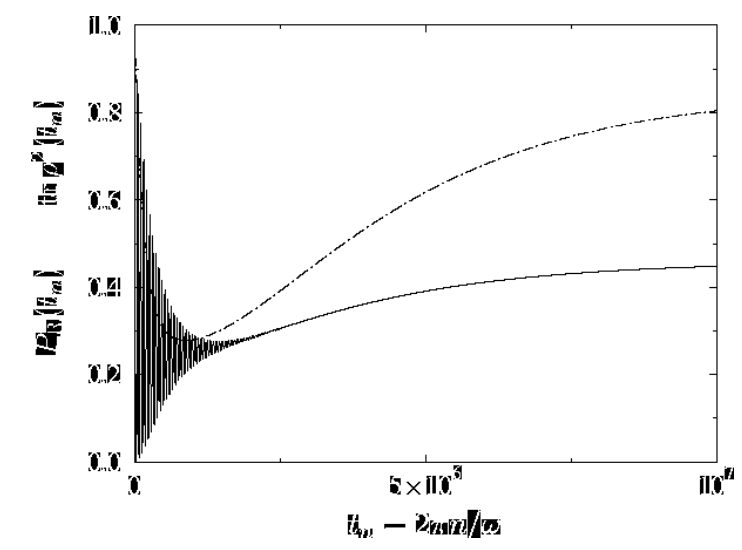

FIG. 9. Time evolution of the return probability $P_{\mathrm{R}}$ (full line) and the coherence function $\operatorname{tr} \rho^{2}$ (dash-dotted) during loss and regain of coherence. The parameter values are as in Fig. $8 \mathrm{~b}$.
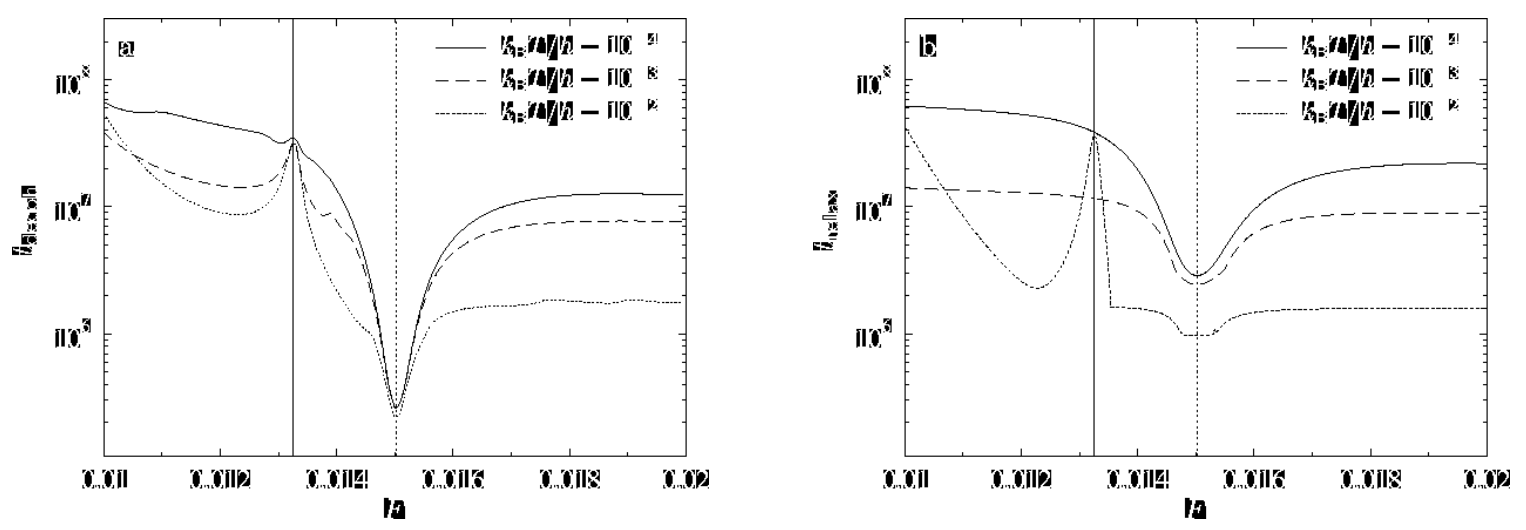
FIG. 10. Time scales of the decay of $\operatorname{tr} \rho^{2}$ (a) and of the relaxation towards the asymptotic solution $(b)$ near the singlet-doublet crossing. Near the exact crossing $(F \approx 0.013$, full vertical line) coherence is stabilized, whereas at the center of the avoided crossing $(F \approx 0.015$, dashed vertical line) the decay of coherence is accelerated. The parameter values are $D / \hbar=4, \omega=0.982$, $\gamma=10^{-6}$, temperature as given in the legend.
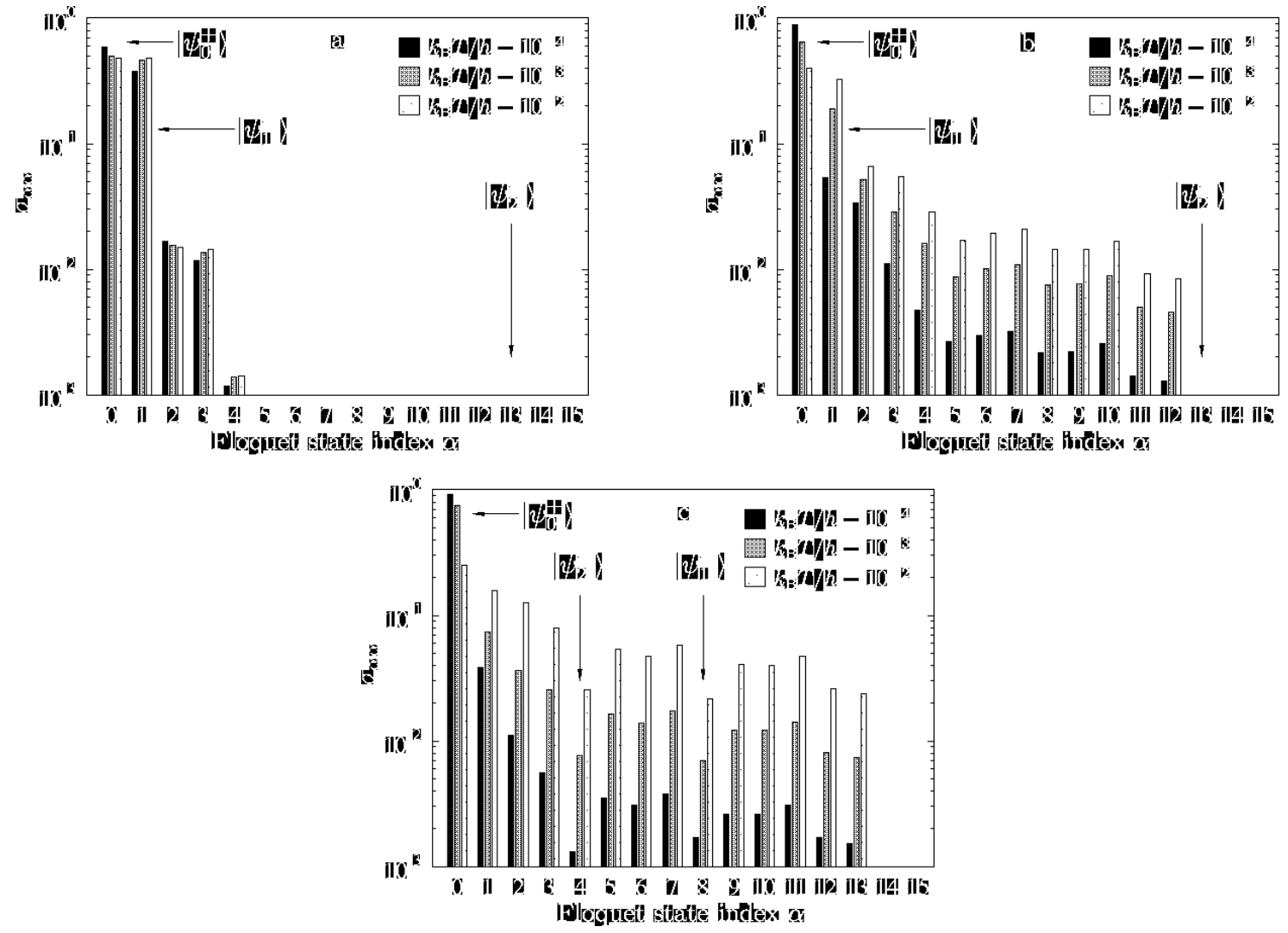

FIG. 11. Occupation probability $\sigma_{\alpha \alpha}$ of the Floquet states $\left|\psi_{\alpha}\right\rangle$ in the long-time limit. The parameter values are $D / \hbar=4, \omega=0.982, \gamma=10^{-6}$, and $F=0.013$ (a), 0.0145 (b), 0.015029 (c), temperature as given in the legend.

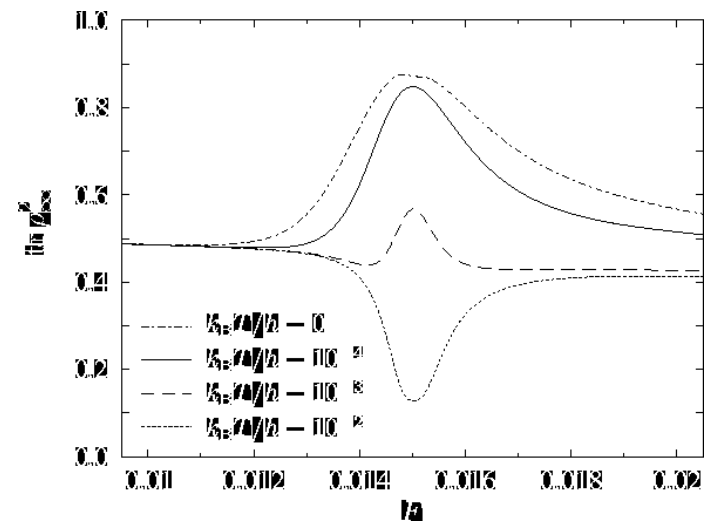


FIG. 12. Coherence of the asymptotic state in the vicinity of a singlet-doublet crossing for different temperatures as given in the legend. The other parameter values are $D / \hbar=4, \omega=0.982$, and $\gamma=10^{-6}$.

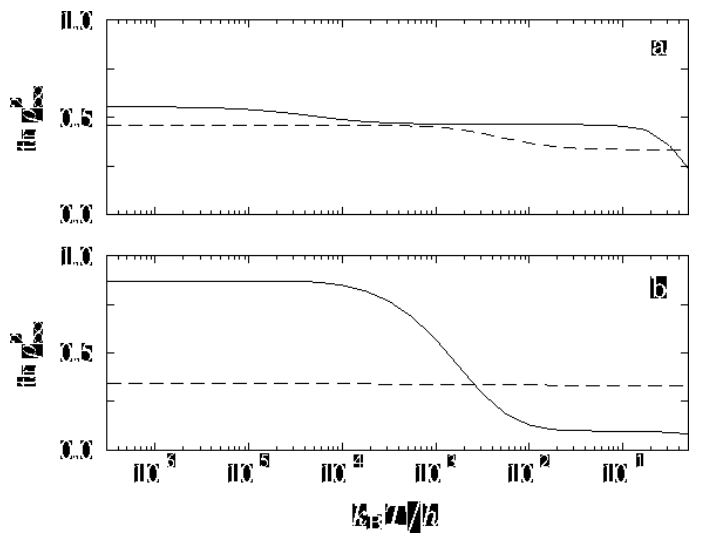

FIG. 13. Coherence of the asymptotic state in the vicinity of a singlet-doublet crossing for $F=0.013$ (a) and $F=0.015029$ (b): exact calculation (full line) compared to the values resulting from a three-level description (dashed) of the dissipative dynamics. The other parameter values are $D / \hbar=4, \omega=0.982$, and $\gamma=10^{-6}$. 